\title{
Special Issue on Body Area Networks
}

\author{
Ilangko Balasingham • Junichi Suzuki • Tao Gu
}

Published online: 10 December 2014

(C) Springer Science+Business Media New York 2014

Foreword: This special issue features three selected papers from the 7th International Conference on Body Area Networks (BodyNets 2012), which took place in Oslo, Norway, from September 24 to 26, 2012. These papers report the recent advances of body area networks, addressing important and emerging research issues and covering novel solutions in design methodologies, algorithms and applications.

The first paper entitled "Disruption-Tolerant Wireless Sensor Networking for Biomedical Monitoring in Outdoor Conditions" leverages disruption-tolerant wireless sensors to monitor the cardiac activity of runners during an outdoor marathon race. Since disruptions often occur in ECG data transmissions from wearable sensor nodes to nearby base stations, which are sparsely available on the marathon route, this paper investigate a store-andforward scheme in which sensor nodes store data in their local caches and attempt to transmit the cached data only when runners pass by base stations. Empirical experiment results are reported regarding data acquisition,

I. Balasingham $(\triangle)$

Oslo University Hospital, University of Oslo, Oslo, Norway

e-mail: ilangko.balasingham@medisin.uio.no

I. Balasingham

Norwegian University of Science and Technology, Trondheim, Norway

\section{J. Suzuki $(\bowtie)$}

University of Massachusetts Boston, Boston, USA

e-mail: jxs@cs.umb.edu

\section{T. Gu}

RMIT University, Melbourne, Australia

e-mail: tao.gu@rmit.edu.au transmission and collection performance with IEEE 802.15.4 (ZigBee), IEEE 802.11 (WiFi) and 3G cellular networks in real marathon races.

The next paper, "Decentralized Positioning Algorithm for Relative Nodes Localization in Wireless Body Area Networks," proposes and evaluates a decentralized localization method that asynchronously estimates the locations of mobile on-body nodes using the information from their direct neighbors. The method utilizes fixedlength links (e.g., between the wrist and elbow) as geometric constraints, which can reduce the number of required online measurements. Through scheduling and censoring, it mitigates error propagation and harmful effects caused by the loss of critical packets. Simulations are carried out to evaluate the proposed method's localization performance under realistic body mobility.

The last paper entitled "A Service-Oriented Architecture for Body Area NanoNetworks with Neuron-based Molecular Communication" studies a neuron-based molecular communication to construct intrabody nanoscale sensor-actuator networks where implanted nanoscale devices are networked through neuronal networks to perform sensing and actuation tasks for biomedical and prosthetic purposes. This paper describes a software architecture to manage and control neuron-based nanonetworks and focuses on a method to artificially grow neurons into particular topology patterns as well as a TDMA-based communication protocol for nanoscale devices to communicate with each other atop neuronal networks. A wet lab experiment and simulations are carried out to validate the proposed network formation method and communication protocol.

The guest editors appreciate reviewers for their efforts in reviewing manuscripts. We are also grateful to Drs. Imrich Chlamtac and Sara Fruner for their significant guidance to edit this special issue. 